\title{
Factors Contributing to Non-Attendance of GI Endoscopic Procedures in a Tertiary Care Center in the Middle East
}

\author{
Israa Hadaib ${ }^{1}$, Pascale Anglade ${ }^{1,2 *}$, Halah Ibrahim ${ }^{3,4}$
}

\begin{abstract}
Introduction: The cancer burden in the Middle East is high and growing. Colorectal cancer (CRC) is the second most common cancer for both men and women in the UAE. Although early diagnosis of malignancy reduces morbidity and increases the survival rates, non-attendance of gastroenterology (GI) endoscopic procedures is a significant global problem, which can lead to delay in cancer diagnosis and treatment. Several factors have been found to contribute to non-attendance behavior, including socioeconomic, cultural, and organizational related barriers. The purpose of this study was to identify factors contributing to non-attendance behavior among outpatients scheduled for GI endoscopic procedures in a tertiary hospital in the United Arab Emirates. We conclude with recommendations that can help in reducing the rate of patient no-shows for GI endoscopic procedures in the region. Methods: In a tertiary medical center in the Middle East, we surveyed patients who did not attend their scheduled GI endoscopic procedures over a period of one year. The questionnaire sought to identify possible reasons for patient's non-attendance. Descriptive measures including means, standard deviation, frequencies, and percentages were used to analyze the demographic characteristics of the study participants. The chi-square test was performed to analyze gender differences. Results: Of 314 outpatients who met study inclusion criteria, 168 agreed to participate (53.5\% response rate). The majority of participants were women ( $\mathrm{n}=96,60.4 \%)$, aged 18 to 73 , with a mean of 42 years. The largest age group was between 35 and $44(\mathrm{n}=46$, $28.9 \%$ ). Approximately equal numbers of non-attendance appointments were scheduled for combined colonoscopy and upper endoscopy (36.3\%), colonoscopy alone (31.3\%), or upper endoscopy alone (31.3\%). The most common causes for cancellation or non-attendance included concerns about the appointment $(35.5 \%)$, inconvenient timing of the appointment $(27.9 \%)$ and changes in medical status $(26.4 \%)$. Gender differences were noted for non-attendance behaviors, with women significantly more likely than men to report feelings of embarrassment (Chi-square 6.261, $\mathrm{df}=1$, $\mathrm{p}=.012$ ). Conclusion: Our study has identified several barriers to patient attendance of endoscopic procedures, as well as opportunities to reduce the rate of patient no-shows, including patient education, scheduling options, and protocols to minimize discomfort and misconceptions around GI endoscopic procedures, particularly accommodating for same gender endoscopists, with the ultimate goal of increasing early cancer screening and prevention.
\end{abstract}

Keywords: Gastroenterology- endoscopy- barriers- non-attendance- United Arab Emirates

Asian Pac J Cancer Prev, 23 (1), 33-37

\section{Introduction}

The cancer burden in the Middle East is high and growing; with models predicting that prevalence will nearly double within the next decade (Allemani et al., 2015). In the United Arab Emirates (UAE), cancer ranks as the third leading cause of death, after cardiovascular disease and trauma (Al Abdouli et al, 2018). Colorectal cancer (CRC) is the second most common cancer for both men and women in the UAE (Al Abdouli et al., 2018). Research shows that early diagnosis of malignancy reduces morbidity and increases the survival rate. There have been substantial government efforts in the UAE to increase cancer awareness and screening. In recent years, large-scale investments in healthcare infrastructure have included the establishment of specialized cancer centers with state-of-the-art diagnostic modalities throughout the country. Additional efforts to improve oncology services include the development of a national cancer registry, population-wide screening programs and funding to support cancer research (Nair et al., 2019). These initiatives are only effective in conjunction with campaigns raising community awareness and participation. Studies have shown that screening programs have low uptakes in Arab populations (Azaiza and Cohen, 2006; Donnelly et al., 2011). Cultural stigma, biases and misinformation 
related to screening can be barriers to early detection, leading to delays in diagnosis and treatment, leaving many patients with cancer in the region seeking care only for more advanced or incurable tumors (Alhurishi et al., 2011; Nair et al., 2018). In a cross-sectional survey of 600 people conducted in 2018 in the UAE, the majority of patients did not perceive CRC to be common and were not aware of any screening procedures. Further, 95\% of survey respondents reported that CRC screening was never recommended to them by a physician (Al Abdouli et al, 2018). Even when screening is recommended, non-attendance rates for gastrointestinal (GI) procedures are high (Al Abdouli et al., 2018; Chang et al., 2015; Laiyemo et al., 2014; Al-Azri et al., 2019). In the United States, GI endoscopy cancellations and non-attendance averaged 21\% in several studies (Chang et al., 2015). There is a large and growing body of research confirming that non-attendance to GI procedure appointments is a global problem (Laiyemo et al., 2014). Several factors have been found to contribute to non-attendance behavior, including socioeconomic, cultural, and organizational related barriers (Chopra and Hookey, 2016; Sahin et al., 2017; Al-Azri et al., 2016). Other authors have suggested that personal factors, such as fear of going through a complex procedure, difficulty of bowel preparation, and previous history of chronic condition, also played a role in non-attendance behavior (Greenspan et al., 2015; Sultan et al., 2017). In our institution, an audit of GI endoscopy appointments scheduled between July 2017 and July 2018 revealed that 1695 patients $(30 \%)$ either canceled or did not show up for their scheduled endoscopy appointments. Given the paucity of published literature on GI procedure non-attendance in the Arab world, and particularly in the Middle East, we sought to identify factors that contributed to our department's high no-show rate. The purpose of this study was to identify factors contributing to nonattendance behavior among outpatients scheduled for GI endoscopic procedures in a tertiary hospital in the UAE. We also sought to measure any associations between demographic factors and non-adherence. Based on our findings, we offer recommendations that can help in reducing the rate of patient no-shows for GI endoscopic procedures.

\section{Materials and Methods}

\section{Setting and Participants}

Cleveland Clinic Abu Dhabi is a 364-bed tertiary care center in Abu Dhabi, the capital and largest emirate in the UAE. The GI department performs approximately 5000 endoscopic procedures annually. Participants included in the study met the following criteria: adults 18 years old or above who were referred from the outpatient services for GI endoscopic procedures but did not attend their appointment (colonoscopy or EGD) between July 1, 2017 and July 1, 2018. Exclusion criteria included patients who did not speak or understand Arabic or English, patients with hearing, mental or cognitive impairment who could not comprehend the survey questions, and individuals who were admitted to the hospital at the time of the data collection. The study was reviewed and approved by the
Cleveland Clinic Abu Dhabi research ethics committee.

\section{Survey Instrument}

The survey instrument was adapted from a questionnaire by Bhise and colleagues (2016), which studied patientreported attributions for missed colonoscopy appointments in two tertiary health care systems in Houston, Texas. The survey was translated from English to Arabic by a back-to-back approach by an independent translator. The survey was piloted for length and clarity with 10 patients, who did not take part in the final study. Through open and closed-ended response items, the questionnaire sought to identify possible reasons for patient's non-attendance for their scheduled GI endoscopy procedure. Demographic information was also collected via the questionnaire or review of the patient's electronic medical record.

\section{Data Collection}

The list of patients who did not attend the scheduled endoscopic procedure appointment was obtained from the Digestive Disease Institute database. Eligible participants were approached via phone call by the primary investigator and invited to participate in the study. Respondents either proceeded directly with the telephone questionnaire or were scheduled for a telephone interview at their convenience. Verbal consent was obtained from participants and all respondents received a study information leaflet via email in their preferred language. Each telephone interview was conducted in Arabic or English as per patient preference and lasted approximately ten to fifteen minutes.

\section{Data Analysis}

De-identified data were statistically analyzed using the IBM Statistical Package for Social Science (SPSS) program version 25.0. Descriptive measures including means, standard deviation, frequencies, and percentages were used to analyze the demographic characteristics of the study participants. The chi-square test was performed to analyze gender differences.

\section{Results}

A total of 314 outpatients who met the study inclusion criteria were called for the telephone interview, with 168 agreeing to be interviewed (53.5\% response rate). Table 1 lists participant demographics. The majority of the participants were women $(n=96,60.4 \%)$. Participant ages ranged from 18 to 73 , with a mean of 42 years. The largest age group was between 35 and $44(n=46,28.9$ $\%)$. Approximately equal numbers of non-attendance appointments were scheduled for combined colonoscopy and upper endoscopy (36.3\%), colonoscopy alone (31.3 $\%$ ), or upper endoscopy alone $(31.3 \%)$.

Self-reported reasons for the scheduled procedures included abdominal pain or GI symptoms (nausea, vomiting, dysphagia or constipation/diarrhea) (97, $60 \%)$, asymptomatic screening purposes $(n=24,15 \%)$, or a history of cancer, polyps, H. pylori infection, rectal bleeding or anemia $(n=42 ; 26 \%)$. For approximately half of the participants $(n=81,50.6 \%)$, this was the first 
DOI:10.31557/APJCP.2022.23.1.33

Factors Contributing to Non-Attendance of GI Endoscopic Procedures in a Tertiary Care Center in the Middle East

Table 1. Demographics of Survey Participants $(\mathrm{N}=160)$

\begin{tabular}{|c|c|c|c|}
\hline Variable & Category & $\mathrm{n}$ & $\% \mathrm{~N}$ \\
\hline \multirow[t]{5}{*}{ Gender } & Male & 63 & 39.6 \\
\hline & Female & 96 & 60.4 \\
\hline & Missing & 1 & 0.6 \\
\hline & 18-24 years old & 13 & 8.2 \\
\hline & 25-34 years old & 35 & 22.0 \\
\hline \multirow[t]{5}{*}{ Age } & 35-44 years old & 46 & 28.9 \\
\hline & 45-54 years old & 37 & 23.3 \\
\hline & 55-64 years old & 19 & 11.9 \\
\hline & $65-75$ years old & 9 & 5.7 \\
\hline & Missing & 1 & 0.6 \\
\hline \multirow[t]{7}{*}{ Level of education } & Doesn't read or write & 6 & 3.8 \\
\hline & Primary Education & 20 & 12.5 \\
\hline & Secondary school & 49 & 30.6 \\
\hline & Associate degree & 17 & 10.6 \\
\hline & Bachelor's degree & 49 & 30.6 \\
\hline & Master`s degree & 15 & 9.4 \\
\hline & Doctoral degree & 4 & 2.5 \\
\hline \multirow[t]{4}{*}{ Marital status } & Single & 32 & 20.0 \\
\hline & Married & 109 & 68.1 \\
\hline & Widowed & 6 & 3.8 \\
\hline & Divorced & 13 & 8.1 \\
\hline \multirow[t]{3}{*}{ Employment Status } & Employed & 83 & 52.5 \\
\hline & Non-Employed & 75 & 47.5 \\
\hline & Missing & 2 & 1.2 \\
\hline
\end{tabular}

endoscopic procedure, while the remainder had undergone a previous procedure within the past 5 years.

The majority of the participants who did not attend their endoscopic appointment $(n=99,62.2 \%)$ were scheduled for a procedure to diagnose an acute symptom, such as abdominal pain, constipation or acid reflux. The most common causes for cancellation or non-attendance included concerns about the appointment (35.5\%), inconvenient timing of the appointment $(27.9 \%)$ and changes in medical status $(26.4 \%)$, primarily resolution of the symptoms for which the procedure was scheduled (Table 2). Gender differences were noted for non-attendance behaviors. Women were significantly more likely than men to report non-attendance because of feelings of embarrassment (Chi-square 6.261, $\mathrm{df}=1$, $\mathrm{p}=0.012$ ). Women also reported more obstacles, including not having a transport or an escort on the day of the procedure (Chi-square $2.388, \mathrm{df}=2, \mathrm{p}=0.061$ ), not clearly understanding bowel prep instructions (Chi-square $0.037, \mathrm{df}=1, \mathrm{p}=.848$ ) and inability in completing the bowel preparation (Chi-square $0.052, \mathrm{df}=1, \mathrm{p}=0.819$ ).

\section{Discussion}

Our study found a high degree of non-attendance for GI procedures in a country with high prevalence of CRC. Primary causes for non-attendance in our patient population mirrored those in previous studies, including concerns about the procedure, appointment
Table 2. Self-Reported Reasons for Not Attending GI Endoscopic Procedure Appointments (N=160)

\begin{tabular}{lcc}
\hline Self-reported reasons for non-attendance & ${ }^{\mathrm{n}}$ & $\% \mathrm{~N}$ \\
\hline Time of the appointment was inconvenient & 43 & 27.9 \\
Waited too long for the appointment & 41 & 26.6 \\
Had a work or family emergency condition & 22 & 13.7 \\
Educational materials for bowel preparation was & 21 & 13.1 \\
not clear & & \\
Did not have an escort at the day of the procedure & 18 & 11.3 \\
Long distance travel to the hospital & 18 & 11.3 \\
Traveling (Not in the country) & 12 & 7.5 \\
Not able to complete the bowel preparation & 11 & 6.9 \\
Does not have a transport & 11 & 6.9 \\
Feeling of embarrassment & 9 & 5.6 \\
Religious restrictions & 8 & 5.0 \\
Required medical or financial clearance & 8 & 5.0 \\
Unaware of their appointment & 7 & 4.4 \\
(didn`t receive reminder) & & \\
Does not know how the procedure will be done & 7 & 4.4 \\
Forgot the appointment & 6 & 3.8 \\
Mixed up the date & 5 & 3.2 \\
Presence of chronic illness & 5 & 3.2 \\
Prefer to consult another doctor & 2 & 1.2 \\
Preferred GI Endoscopy with a female doctor & 2 & 1.2 \\
\hline
\end{tabular}

scheduling issues and symptom resolution. The majority of respondents reported two major concerns as their reason for non-attendance, specifically concerns regarding how the procedure would be done and difficulty in preparation for the procedure. These results are comparable with those of the study by Wong et al., (2013), which also found that concerns about the procedure were significant barriers for patients scheduled for colonoscopy. We are concerned that a substantial number of patients scheduled for surveillance or screening purposes $(26 / 69,37.7 \%)$ did not attend their appointment. This result is inconsistent with a US study, which showed a higher attendance rate among patients who were scheduled for their initial screening colonoscopy (74\%) or a surveillance colonoscopy (78\%) (Gardiner, 2016). Providing detailed patient information, including audiovisual information, can help to counteract misinformation and allay patient fears. In addition, proper training of healthcare providers and support staff is important to inform patients about their diagnoses and necessary work up and treatments. In one study, patients who received screening recommendations before their colonoscopy appointment were 3.7 times more likely to complete the procedure (Nagelhout et al., 2017). As such, patient education materials highlighting the benefits of cancer screening are essential. Our findings also suggest that women patients in our population may need additional education and support.

As the majority of study participants were scheduled due to acute GI symptoms, which generally last from seven to ten days, over a quarter of the patients did not attend their scheduled appointment because their health condition improved. Similar results were noted in a cohort 
of 129,549 colonoscopies, where patients with nonspecific abdominal symptoms had a lower completion rate for colonoscopy, as compared to other indications (Gupta et al., 2010). Studies have shown that time between the scheduled and actual appointment, known as lead time, of more than two weeks significantly increases the probability of appointment non-attendance (Navarro et al., 2017). Efforts to minimize this lead time can be effective in increasing appointment attendance rates. In one study, $11 \%$ of patients admitted that they would not undergo a colonoscopy even if they had symptoms of CRC (Zapatier et al., 2011). To maximize compliance and attendance adherence in this patient population, healthcare providers should introduce the available screening options and allow patients to select the test they feel most appropriate for them.

Patient non-attendance not only affects the health of the individual patient due to delay in diagnosis or treatment, but also adversely impacts prospective patients by limiting their ability to schedule timely appointments. It also negatively affects space and manpower utilization and revenue generation, thereby, causing financial constraints to healthcare organizations (Chang et al., 2015; Hwang et al., 2015). As such, minimizing non-attendance rates can have substantial clinical and organizational benefits. Overbooking has been adopted by many healthcare organizations to mitigate underutilization due to non-attendance. Models have been developed using patient lateness and no-show rates and percentage overbooking on patient waiting time and doctors' utilization and overtime (Satiani et al., 2009). For GI endoscopic procedures, organization-specific metrics should be employed to ensure that percentage overbooking mitigates high no-show rates without increasing patient wait times or staff overtime. The Stochastic Mathematical Overbooking Model (SMOM) has been successfully used, with one study showing an average profit increase of $43.72 \%$ for 59 clinics after implementation of the model (Kim and Giachetti, 2006).

It is notable that women in our study reported a higher degree of embarrassment as a reason for non-attendance. In fact, social, religious and cultural factors have been shown to affect patient healthcare decisions. In our institution, we have employed a few simple yet effective strategies to overcome these barriers. For example, to minimize embarrassment, we offer disposable garments with openings in the back that can be worn under hospital gowns, thereby limiting areas of the body exposed during colonoscopy. We also deliberately wait until adequate sedation has been achieved before uncovering patients and ensure that patients are completely covered while in transit to and from the procedure room. As studies have shown that many female patients prefer same gender endoscopists when scheduling procedures (Zapatier et al., 2011; Shah et al., 2011; Varia et al., 2014), we routinely ask our patients if they prefer a specific gender endoscopist. The overwhelmingly gender-concordant preferences expressed by our female patients have driven significant change, including the initiation of a "For Women By Women" endoscopy service, with exclusively female caregivers from admission to discharge.

Limitations of this study include single institution and small sample size. Nonetheless, our findings confirm those of previous international studies and suggest that mitigation strategies employed in Western countries may also be effective in our setting. Accordingly, our successful interventions may be generalizable to endoscopy practices in the region and worldwide.

In conglusion as the cancer rates climb in the UAE, it is important to fully understand challenges to cancer screening. Our study has identified several barriers to patient attendance of endoscopic procedures, as well as opportunities to reduce the rate of patient no-shows, including patient education, scheduling options, and protocols to minimize discomfort and misconceptions around GI endoscopic procedures, particularly accommodating for same gender endoscopists. Future studies should assess the efficacy of these interventions, with the ultimate goal of minimizing non-attendance rates and improving patient outcomes and healthcare efficiency.

\section{Author Contribution Statement}

Israa Hadaib: MSc conceived the study and collected the data; Pascale Anglade: MD, MBA analyzed the data and drafted the manuscript; Halah Ibrahim: MD, MEHP analyzed the data and drafted the manuscript. All authors read and reviewed the final manuscript.

\section{Acknowledgements}

Study approval

the study was reviewed and approved by the Cleveland Clinic Abu Dhabi research ethics committee.

Availability of data

data is available from the authors upon written request.

\section{Conflict of interest}

there was no conflict of interest.

\section{References}

Al Abdouli L, Dalmook H, Akram Abdo M, et al (2018). Colorectal cancer risk awareness and screening uptake among Adults in the United Arab Emirates. Asian Pac J Cancer Prev, 19, 2343-49.

Al-Azri M, Al-Maskari A, Al-Matroushi S, et al (2016). Awareness of cancer symptoms and barriers to seeking medical help among adult people attending primary care settings in Oman. Health Serv Res Manag Epidemiol, 3, 2333392816673290.

Al-Azri M, Al-Kindi J, Al-Harthi T, et al (2019). Awareness of stomach and colorectal cancer risk factors, symptoms and time taken to seek medical help among public attending primary care setting in Muscat Governorate, Oman. J Cancer Educ, 34, 423.

Alhurishi S, Lim JN, Potrata B, West R (2011). Factors influencing late presentation for breast cancer in the Middle East: a systematic review. Asian Pac J Cancer Prev, 12, 1597-600.

Allemani C, Weir HK, Carreira H, et al (2015). Global 
surveillance of cancer survival 1995-2009: analysis of individual data for $25,676,887$ patients from 279 populationbased registries in 67 countries (CONCORD-2). Lancet, 385, 977-1010

Azaiza F, Cohen M. (2006). Health beliefs and rates of breast cancer screening among Arab women. $J$ Womens Health (Larchmt), 15, 520-30.

Bhise V, Modi V, Kalavar A, et al (2016). Patient-reported attributions for missed colonoscopy appointments in two large healthcare systems. Dig Dis Sci, 61, 1853-61.

Chang JT, Sewell JL, Day LW (2015). Prevalence and predictors of patient no-shows to outpatient endoscopic procedures scheduled with anesthesia. BMC Gastroenterol, 15, 123.

Chopra D, Hookey LC (2016). Comorbid illness, bowel preparation, and logistical constraints are key reasons for outpatient colonoscopy nonattendance. Can J Gastroenterol Hepatol, 2016, 2179354.

Donnelly TT, Al-Khater A-H, Al-Kuwari M, et al (2011). Study exploring breast cancer screening practices amongst Arabic women living in the state of Qatar. Avicenna, 2011, 1.

Gardiner KL (2016). Barriers to colorectal cancer screening in people obtaining care From community mental health agencies. Walden Dissertations and Doctoral Studies. 2653.

Greenspan M, Chehl N, Shawron K, et al (2015). Patient non-adherence and cancellations are higher for screening colonoscopy compared with surveillance colonoscopy. Dig Dis Sci, 60, 2930-6.

Gupta M, Holub JL, Eisen G (2010). Do indication and demographics for colonoscopy affect completion? A large national database evaluation. Eur J Gastroenterol Hepatol, 22, 620-7.

Hwang JH, Konda V, Abu Dayyeh BK, et al (2015). Endoscopic mucosal resection. Gastrointest Endosc, 82, 215-26.

Kim S, Giachetti RE (2006). A stochastic mathematical appointment overbooking model for healthcare providers to improve profits. IEEE Trans Syst Man Cybern a Syst Hum, 36, 1211-9.

Laiyemo AO, Williams CD, Burnside C, et al (2014). Factors associated with attendance to scheduled outpatient endoscopy. Postgrad Med J, 90, 571-5.

Nagelhout E, Comarell K, Samadder NJ, Wu YP (2017). Barriers to colorectal cancer screening in a racially diverse population served by a safety-net clinic. J Community Health, 42, 791-6.

Nair SC, Jaafar H, Jaloudi M, et al (2018). Supportive care needs of multicultural patients with cancer in the United Arab Emirates. Ecancermedicalscience, 29, 838.

Nair SC, Hassen WA, Sreedharan J, Qawasmeh K, Ibrahim H (2019). Comparison of perceptions of unmet supportive care needs between cancer patients and their oncologists. Support Care Cancer, 27, 4229-35.

Navarro MJ, LaPiene B, Sivak S (2017). Wait times less than 2 weeks minimize no-show rates in cardiology practices. $\mathrm{Am}$ J Med Qual, 32, 684.

Sahin MK, Aker S, Arslan HN (2017). Barriers to colorectal cancer screening in a primary care setting in Turkey. J Community Health, 42, 101-8.

Satiani B, Miller S, Patel D (2009). No-show rates in the vascular laboratory: analysis and possible solutions. J Vasc Interv Radiol, 20, 87-91.

Shah DK, Karasek V, Gerkin RD, et al (2011). Sex preferences for colonoscopists and GI physicians among patients and health care professionals. Gastrointest Endosc, 74, 122-7.e2.

Sultan S, Partin MR, Shah P, et al (2017). Barriers and facilitators associated with colonoscopy completion in individuals with multiple chronic conditions: a qualitative study. Patient Prefer Adherence, 11, 985-94.

Varia A, Patel MK, Tanikella R, et al (2014). Gender preference for the endoscopist among Hispanics: the results of a prospective study. J Immigr Minor Health, 16, 990-3.

Wong RK, Wong ML, Chan YH, et al (2013). Gender differences in predictors of colorectal cancer screening uptake: a national cross sectional study based on the health belief model. BMC Public Health, 13, 677.

Zapatier JA, Kumar AR, Perez A, et al (2011). Preferences for ethnicity and sex of endoscopists in a Hispanic population in the United States. Gastrointest Endosc, 73, 89-97.e974.

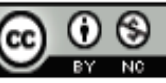

This work is licensed under a Creative Commons AttributionNon Commercial 4.0 International License. 